\title{
Biological Efficacy of Streptomyces sp. Strain BN1 against the Cereal Head Blight Pathogen Fusarium graminearum
}

\author{
Boknam Jung ${ }^{1}$, Sook-Young Park ${ }^{2}$, Yin-Won Lee ${ }^{2}$ and Jungkwan Lee ${ }^{1 *}$ \\ ${ }^{\prime}$ Department of Applied Biology, Dong-A University, Busan 604-714, Korea \\ ${ }^{2}$ Department of Agricultural Biotechnology and Center for Fungal Pathogenesis, Seoul National University, Seoul 151-921, Korea \\ (Received on July 24, 2012; Revised on October 11, 2012; Accepted on October 14, 2012)
}

Fusarium head blight (FHB) caused by the filamentous fungus Fusarium graminearum is one of the most severe diseases threatening the production of small grains. Infected grains are often contaminated with mycotoxins such as zearalenone and trichothecences. During survey of contamination by $\mathrm{FHB}$ in rice grains, we found a bacterial isolate, designated as $\mathrm{BN1}$, antagonistic to $F$. graminearum. The strain BN1 had branching vegetative hyphae and spores, and its aerial hyphae often had long, straight filaments bearing spores. The 16S rRNA gene of BN1 had $100 \%$ sequence identity with those found in several Streptomyces species. Phylogenetic analysis of ITS regions showed that BN1 grouped with $S$. sampsonii with $77 \%$ bootstrap value, suggesting that $B N 1$ was not a known Streptomyces species. In addition, the efficacy of the BN1 strain against $F$. graminearum strains was tested both in vitro and in vivo. Wheat seedling length was significantly decreased by $\boldsymbol{F}$. graminearum infection. However, this effect was mitigated when wheat seeds were treated with $\mathrm{BN1}$ spore suspension prior to $F$. graminearum infection. BN1 also significantly decreased FHB severity when it was sprayed onto wheat heads, whereas BN1 was not effective when wheat heads were point inoculated. These results suggest that spraying of BN1 spores onto wheat heads during the wheat flowering season can be efficient for plant protection. Mechanistic studies on the antagonistic effect of BN1 against $F$. graminearum remain to be analyzed.

Keywords : biocontrol agent, fusarium head blight, Fusarium graminearum, Streptomyces

Fusarium head blight (FHB) is one of the most severe diseases threatening the production of major cereal crops including wheat, barley, and rice. Infected grains are often contaminated with mycotoxins such as zearalenone and trichothecences that cause mycotoxicosis in humans and animals (Desjardins, 2006; Lee et al., 2009). Although

\footnotetext{
*Corresponding author.

Phone) +82-51-200-7554, FAX) +82-51-200-7505

E-mail) jungle@dau.ac.kr
}

several fungal species of the Fusarium genus can cause FHB, the ascomycete fungus $F$. graminearum (telomorph: Gibberella zeae) is a major causal agent for FHB in Asia and North America (Leslie and Summerell, 2006). In Korea, $F$. graminearum recently caused severe yield losses in wheat, barley, rice, and maize (Lee et al., 2009, 2010, 2012; Ryu et al., 2011). In addition to yield losses and mycotoxin contamination through disease, planting infected seeds can cause root rot and seedling blight (Leslie and Summerell, 2006).

Despite the toxigenic and pathogenic importance of FHB, there are no FHB resistant cultivars and there is only limited information on biological agents against FHB. Fungicide application has shown partial efficacy for FHB control. Azole-type fungicides, one of the most important classes of systemic site-specific fungicides, reduce grain infection and trichothecene contamination (Pirgozliev et al., 2003). The fungicides inhibit cytochrome P450 sterol $14 \alpha-$ demethylase, resulting in the disturbance of fungal membrane integrity (Buchenauer, 1987). However, resistance to azole fungicides occurs in many plant pathogens including F. graminearum (Becher et al., 2010), limiting their widespread application and requiring the development of other approaches including resistant cultivars, cultivation methods, and biological control agents.

One of the most plentiful natural resources for the development of biological agents against plant pathogens are the Gram-positive Actinobacteria, which have high guanine and cytosine content in their genome. Stretomyces, which has highly differentiated branched mycelia, is the largest genus of Actinobacteria (Ventura et al., 2007). Many species belonging to this genus produce diverse secondary metabolites, some of which have antibiotic effects and affect soil microbial communities. In agriculture, the genus Streptomyces plays important roles in increasing soil fertility, degrading organic matter, and controlling pathogens. For example, this genus is a major soil decomposer that provides natural fertilizers for plants. Additionally, blasticidin $\mathrm{S}$ produced by $S$. griseochromogenes is effective for rice blast disease caused by the filamentous fungus Magnaporthe 
oryzea, and polyoxin produced by $S$. asoenosis is used for the control of bacterial rice sheath blight and fungal leaf spot (Watve et al., 2001). The diversity of Streptomyces species in soil provides numerous possibilities to explore potential biological control agents and new secondary metabolites that have antagonistic effects against plant pathogens.

Several filamentous fungi, yeast, and bacteria have antagonistic effects against $F$. graminearum (Luz et al., 2003; Pirgozliev et al., 2003) such as in vitro antifungal activity (Chan et al., 2003; Xue et al., 2009), the reduction of disease severity (Nourozian et al., 2006; Stockwell et al., 2001; Xue et al., 2009), systemic movement within infected spikes (Yuen et al., 2003), mycotoxin accumulation (Dawson et al., 2004), and pathogen survival (Bujold et al., 2001). Although many studies have focused on the development of biological agents against this disease, only a few biological agents including Bacillus subtilis and Cryptococcus laurentii strains have been evaluated under diverse growing conditions and developed as commercial biological agents (Pryor et al., 2007; Zhang et al., 2005). Therefore, more biological agents from natural environmental conditions need to be found and applied as alternatives to chemical approaches for control of FHB.

We found a bacterial colony that inhibited the mycelial growth of $F$. graminearum, suggesting that the bacterial strain can be developed as a biological agent against $F$. graminearum. In the present study, we identified the strain based on the sequences of the 16S rRNA gene and internal transcribed space (ITS) region as well as its morphological characteristics, and tested the efficacy of the strain against $F$. graminearum both in vitro and in vivo. Wheat heads treated with the BN1 strain spore suspension had significantly reduced FHB disease severity caused by F. graminearum, showing that the BN1 strain was able to control FHB.

\section{Materials and Methods}

Isolation and identification of the bacterial strain BN1. A bacterial colony was isolated from rice kernels that were placed on potato dextrose agar (PDA; Leslie and Summerell, 2006) to purify $F$. graminearum isolates. The bacterial colony was grown on lysogeny broth (LB; Sambrook and Russell, 2001) agar plate. To obtain chromosomal DNA, the strain, designated as BN1, was incubated in $3 \mathrm{ml}$ of $\mathrm{LB}$ broth for $48 \mathrm{~h}$ at $28^{\circ} \mathrm{C}$ in an orbital shaker (200 rpm). Chromosomal DNA was isolated using the NucleoSpin columns (Macherey-Nagel, Duren, Germany) following manufacturer's instruction. The primers ITS-38R (5'-CCG GGTTTCCCCATTCGG-3') and ITS-72F (5'-TGCGGCTG GATCTCCTT-3') (Normand et al., 1996) were used for amplification of the ITS region, and 16S-530F (5'-TGACT GACTGAGTGCCAGCMGCCGCGG-3') and 16S-1494R (5'-TGACTGACTGAGGYTACCTTGTTACGACTT-3') primers were used for amplification of the 16S rRNA gene (Borneman and Triplett, 1997). The amplified 16S rRNA gene was directly purified using MEGAquick-spin ${ }^{\mathrm{TM}}$ Total Fragment DNA Purification Kit (iNtRON Biotechnology, Sungnam, Korea) and directly sequenced. In the case of ITS region, the purified PCR product was cloned in pGEMT Easy vector (Promega, Madison, WI), and the clone were sequenced. All the amplified DNA fragments and plasmid clones were sequenced using an automated 3730XL DNA sequencer (ABI, Tokyo, Japan). The sequences were used for a BlastN search in the NCBI GenBank database (http:// blast.ncbi.nlm.nih.gov/Blast.cgi).

Phylogenetic analysis. ITS sequences from the 13 Streptomyces spp. were retrieved from GenBank database. The sequences were aligned using the MEGA4.1 software with default parameters (Tamura et al., 2007), and trimmed manually at the same position that only includes ITS region. The ITS sequence of Actinomyces sp. strain F0330 (GenBank accession number NZ ACTB01000202) was used as the outgroup for the phylogenetic anlaysis. A phylogenetic tree was constructed using the neighbor-joining method (Saitou and Nei, 1987) in the MEGA4.1 software with the following parameters: complete deletion of gaps, Kimura-2, both transitions and transversions substitutions included, and 2,000 bootstrap replicates.

Preparation of inoculum. The bacterial strain BN1 was incubated in $30 \mathrm{ml}$ of $\mathrm{LB}$ for $72 \mathrm{~h}$ at $28^{\circ} \mathrm{C}$ in an orbital shaker $(200 \mathrm{rpm})$ to induce spore production. To harvest BN1 spores, the culture was filtered with two layers of miracloth (Calbiochem, La Jolla, CA, USA) and the filtrates were centrifuged at $10,000 \mathrm{~g}$. The spores were washed twice with distilled water and resuspended with $0.1 \%$ (v/v) Tween 20 solution $\left(10^{6}\right.$ spores $\left./ \mathrm{ml}\right)$. To induce spore production of F. graminearum strain JL001, the strain was incubated in $30 \mathrm{ml}$ of carboxymethylcellulose (CMC) medium as previously described (Capellini and Peterson, 1965). The cultures were also filtered with two layers of miracloth and spores were harvested by centrifugation at $10,000 \mathrm{~g}$. Spores were washed twice with distilled water and diluted to a final $105 / \mathrm{ml}$ concentration in $0.1 \%(\mathrm{v} / \mathrm{v})$ Tween 20 solution.

Antifungal activity assay of the bacterial strain BN1 on diverse media. The biological efficacy of the BN1 strain against $F$. graminearum was tested on four different media including PDA, yeast malt agar (YMA; Harris, 2005), complete medium (CM), and minimal medium (MM; Leslie 
and Summerell, 2006). A sterilized paper disk was placed on one side of each 9-cm Petri-dish and $20 \mu \mathrm{l}$ of BN1 spore suspension $\left(10^{6} \mathrm{spores} / \mathrm{ml}\right)$ or distilled water dropped onto the paper disk. An agar block ( $5 \mathrm{~mm}$ diameter) taken from the margin of a 5-day-old PDA culture of $F$. graminearum JL001 strain was placed on the other side of each plate. The inoculated plates were incubated at $25^{\circ} \mathrm{C}$ for five days. To check the inhibitory effect of the bacterial strain against the fungal strain, distance between the bacterial colony and fungal colony was measured.

Efficacy assays of BN1 against $\boldsymbol{F}$. graminearum on wheat seedlings. Wheat cultivar Eunpamil, which is susceptible to FHB, seeds were soaked in distilled water or BN1 spore suspension $\left(10^{6}\right.$ spores $\left./ \mathrm{ml}\right)$ for $1 \mathrm{~h}$ in an orbital shaker $(100$ rpm). The wheat seeds were dried on a clean hood for $2 \mathrm{~h}$ and then each ten seeds were planted in pots. Before covering the seeds with soil, one $\mathrm{ml}$ of $F$. graminearum conidia suspension $\left(10^{5}\right.$ spores $\left./ \mathrm{ml}\right)$ was sprayed onto the seeds. The pots were placed in a greenhouse for 2 weeks and the length of each seedling was measured to determine the efficacy of BN1 against $F$. graminearum during wheat seedling growth. All experiments were repeated three times and the Tukey test using SPSS 12.0 software (SPSS Inc., Chicago, IL, USA) was performed to determine the significant differences $(P<0.05)$ among the mean values of samples.

Efficacy assays of BN1 against $\boldsymbol{F}$. graminearum on wheat heads. The efficacy of BN1 against $F$. graminearum on wheat heads was tested using two inoculation methods including spraying and point inoculation. For the spraying inoculation, one $\mathrm{ml}$ of $F$. graminearum spore suspension $\left(10^{5}\right.$ spores $\left./ \mathrm{ml}\right)$ or one $\mathrm{ml}$ of mixed spore suspension $\left(10^{5}\right.$ of F. graminearum spores and $10^{6}$ of BN1 spores) were sprayed onto each head (at the mid-anthesis stage) of the wheat cv. Eunpamil. For the point inoculation, $10 \mu \mathrm{l}$ of $F$. graminearum spore suspension $\left(10^{5}\right.$ spores $\left./ \mathrm{ml}\right)$ or $10 \mu \mathrm{l}$ of mixed spore suspension $\left(2 \times 10^{5}\right.$ of $F$. graminearum spores $/ \mathrm{ml}$ and $2 \times 10^{6}$ of BN1 spores $/ \mathrm{ml}$ ) were injected into a center spikelet of a wheat head at mid-anthesis. For the negative controls, wheat heads were treated with sterilized distilled water only or BN1 spore suspension without $F$. graminearum spore suspension. The inoculated wheat head was wrapped with a plastic bag and maintained for 2 days, after which the plastic bags were removed from the infected wheat heads. The plants were placed and grown in a greenhouse and the number of wheat kernels was counted after 2 weeks.

\section{Results}

Identification of the bacterial strain BN1. During survey of rice kernels infected by FHB, we isolated a bacterial colony, named BN1. The bacterial strain BN1 produced branched hyphae and spores both on solid and in liquid LB, $\mathrm{CM}$, and YMA media. Aerial hyphae on solid media often had long, straight filaments bearing spores. These morphological characteristics suggest that this strain belongs to the genus Streptomyces. The 16S rRNA primers amplified a $956 \mathrm{bp}$ fragment from genomic DNA of the BN1 strain and the amplified fragment had 100\% sequence identity with the 16S rRNA gene found in several Streptomyces spp. including $S$. albidoflavus, $S$. gougerotii, $S$. rutgersensis, $S$. diastaticus, and $S$. rutgersensis. This result confirmed that the BN1 strain belonged to the genus Streptomyces, but the 16S rRNA was not diverse enough to identify the species level in this genus. ITS-38R and ITS-72F primers amplified a 474 bp fragment. We excluded the 16S and 23S rRNA regions from the amplified sequence and finally used a 321 bp ITS region to compare the ITS sequences to other known bacterial species. The ITS region of the BN1 strain showed the highest identity (96\%) with that of $S$. sampsonii, and the phylogenetic tree showed that BN1 grouped with $S$. sampsonii $(77 \%$ BS) (Fig. 1). The alignment and trimming of ITS region sequences produced $331 \mathrm{bp}$ with gaps, of which 177 sites $(53.5 \%)$ were conserved and 150 sites $(45.3 \%)$ were variable. The overall mean distance of the applied sequences was 0.246 . In the NJ tree, all the 13 Streptomyces spp. and Streptomyces sp. BN1 showed distinct differences (Fig. 1). Eleven sites (3.4\%) were variable among the 320 sites of ITS region sequences between between BN1 and S. sampsonii (data not shown).

In vitro efficacy of the BN1 strain against $F$. graminearum. The in vitro efficacy level of BN1 strain against $F$.

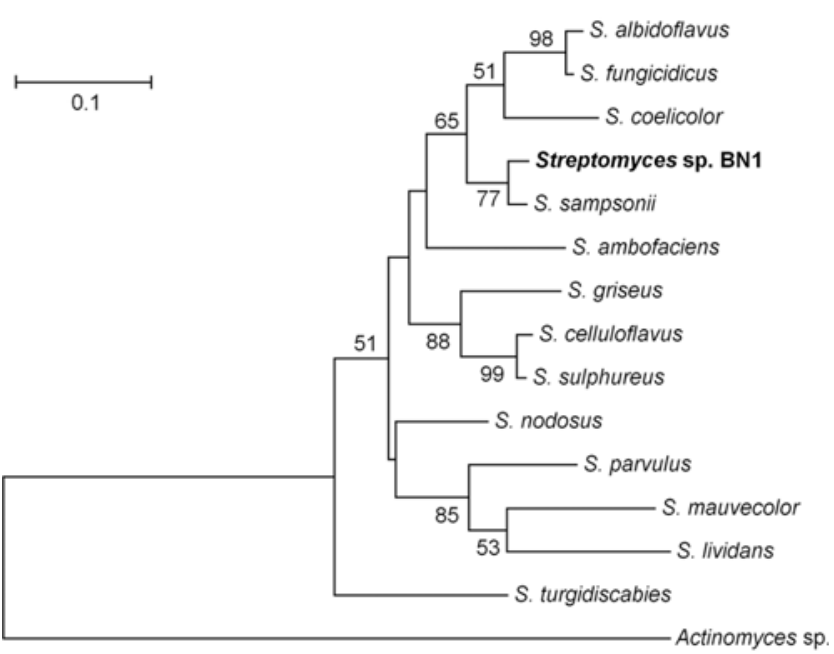

Fig. 1. Phylogenetic analysis of ITS regions identified from the Streptomyces sp. strain BN1 compared to other known Actinobacteria species. The sequences were aligned with ClustalW, and the MEGA software version 4.0 was used to perform a 2000 bootstrap phylogenetic analysis using the neighbor-joining method. 


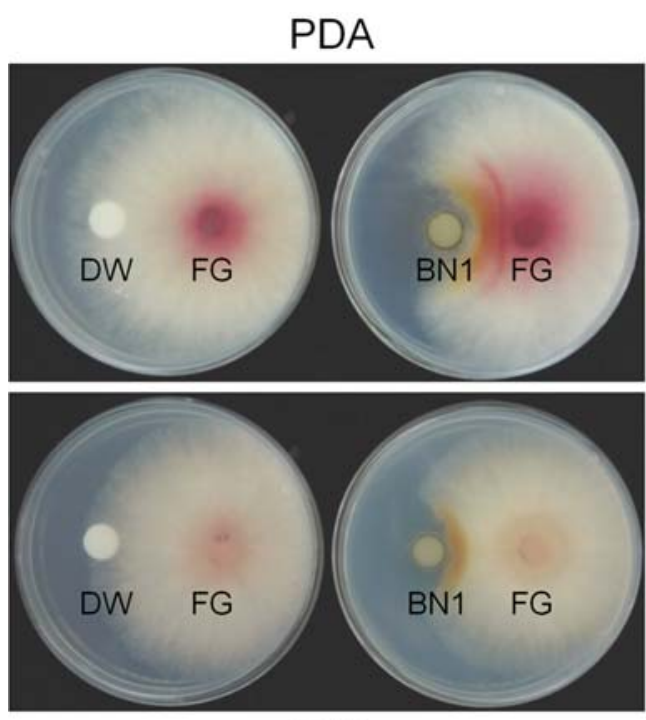

$\mathrm{CM}$
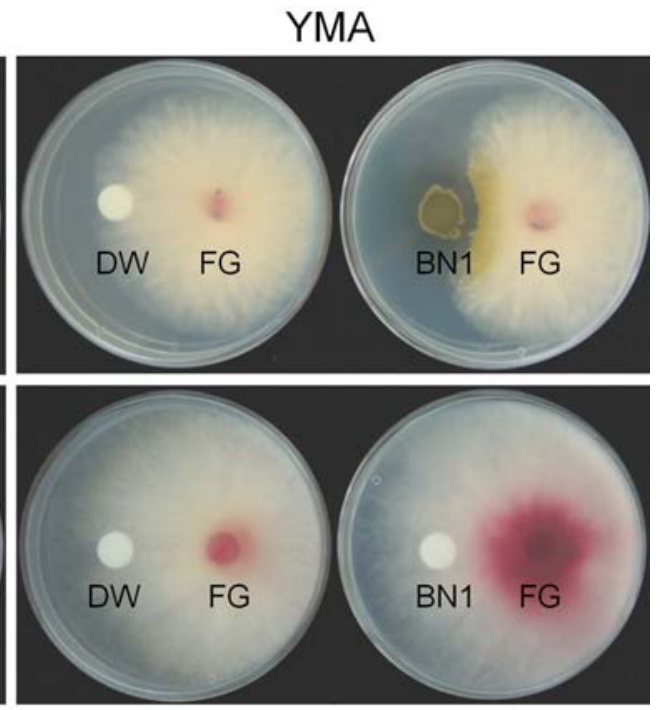

MM

Fig. 2. Efficacy of the Streptomyces sp. strain BN1 against mycelial growth of F. graminearum strain JL001 (FG) on diverse media. Twenty $\mu \mathrm{l}$ of distilled water (DW) or BN1 spore suspension $\left(10^{6} \mathrm{spores} / \mathrm{ml}\right)$ were dropped onto a paper disk on one side of each plate, and $5 \mathrm{~mm}$ diameter agar blocks taken from the margin of a 5-day-old culture of $F$. graminearum strain JL001 were placed on the other side of each plate. The plates were incubated at $25^{\circ} \mathrm{C}$ and photographs were taken 5 days after incubation. PDA, potato dextrose agar; YMA, yeast malt agar; CM, complete medium; MM, minimal medium.

graminearum was slightly different based on the media tested. The BN1 strain mostly inhibited $F$. graminearum mycelia growth on YMA, PDA, and CM, but not on MM (Fig. 2). The level of inhibition in the media was dependent on the extent of BN1 colonization on each medium. On MM, BN1 strain did not grow at all, resulting in the nonefficacy of BN1 against $F$. graminearum on this medium (Fig. 2). When the BN1 strain was first inoculated in PDA, YMA, and CM to allow colonization in the media, followed by the inoculation of $F$. graminearum 3 days later, the inhibition levels were higher than co-inoculation of BN1 and $F$. graminearum (data not shown).

Efficacy of BN1 on wheat seed germination and seedling growth. Wheat seeds soaked in or sprayed with F. graminearum spore suspension had a similar germination rate as wheat seeds that were not treated with $F$. graminearum spore suspension. All experimental conditions had 70$80 \%$ wheat seed germination rate. However, seedling length was significantly decreased in the plants inoculated with $F$. graminearum spore suspension compared to that of non-inoculated plants (Table 1). Seedling length of the wheat seeds treated with BN1 spore suspension before $F$. graminearum inoculation was not significantly different from the non-inoculated plants (Table 1).

Efficacy of BN1 against $\boldsymbol{F}$. graminearum on wheat heads. When the spore suspension was sprayed onto wheat heads,
Table 1. Efficacy of Streptomyces sp. strain BN1 against $F$. graminearum

\begin{tabular}{lccc}
\hline \hline & \multicolumn{2}{c}{$\begin{array}{c}\text { Percentage of kernels with FHB } \\
\text { symptoms }\end{array}$} & $\begin{array}{c}\text { Length of } \\
\text { seedlings }\end{array}$ \\
\cline { 2 - 3 } & \multicolumn{2}{c}{$\begin{array}{c}\text { Spray method } \\
\text { Point inoculation }\end{array}$} & \\
\hline Distilled water & $0^{\mathrm{A}, \mathrm{b}}$ & $0^{\mathrm{A}}$ & $12.3^{\mathrm{A}}$ \\
$\mathrm{BN} 1$ & $0^{\mathrm{A}}$ & $0^{\mathrm{A}}$ & $12.0^{\mathrm{A}}$ \\
$\mathrm{FG}$ & $77^{\mathrm{C}}$ & $65^{\mathrm{B}}$ & $10.3^{\mathrm{B}}$ \\
$\mathrm{BN} 1+\mathrm{FG}$ & $45^{\mathrm{B}}$ & $69^{\mathrm{B}}$ & $11.6^{\mathrm{A}}$ \\
\hline
\end{tabular}

${ }^{\mathrm{a}}$ Wheat heads were inoculated by either spray or point inoculation methods. For the spray method, $1 \mathrm{ml}$ of spore suspension was sprayed onto heads of the wheat cultivar 'Eunpamil', which is susceptible to FHB, at the mid-anthesis stage. For the point inoculation method, $10 \mu \mathrm{l}$ of spore suspension was injected into a center spikelet of the wheat head at mid-anthesis. The wheat heads were wrapped with plastic bags to maintain humidity for 2 days, after which the plastic bags were removed from the infected wheat heads. The plants were grown in a greenhouse and the number of wheat kernels was counted after 2 weeks. BN1, Streptomyces sp. strain BN1; FG, Fusarium graminearum strain JL001.

${ }^{\text {b } V a l u e s ~ w i t h i n ~ a ~ c o l u m n ~ w i t h ~ d i f f e r e n t ~ l e t t e r s ~ a r e ~ s i g n i f i c a n t l y ~ d i f f e r-~}$ ent according to Tukey's test $(P<0.05)$ among the mean values of samples. Ten wheat heads were inoculated for each treatment.

${ }^{\mathrm{c}}$ Wheat seeds were soaked in spore suspension for $1 \mathrm{~h}$ before planting into pots. The pots were placed in a greenhouse for 2 weeks and the length of each seedling was measured to check the efficacy of BN1 against $F$. graminearum.

the F. graminearum strain JL001 produced typical FHB symptoms after a 14-day inoculation, while BN1 did not trigger any disease symptoms (Fig. 3 and Table 1). When 

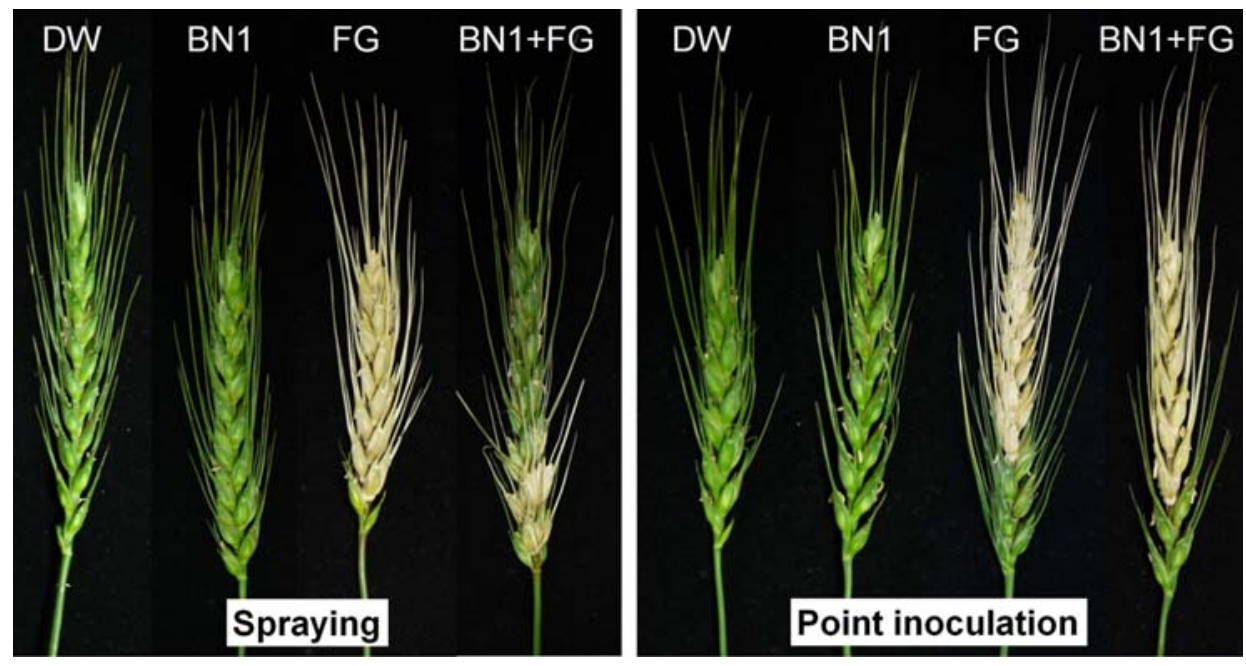

Fig. 3. Efficacy of the Streptomyces sp. strain BN1 against F. graminearum strain JL001 (FG) on wheat heads. Wheat heads were inoculated using the spraying or point inoculation method. For negative controls, wheat heads were treated with either distilled water (DW) or the BN1 spore suspension. Each wheat head was wrapped with a plastic bag to maintain humidity for 2 days, after which the plastic bags were removed from the infected wheat heads. The plants were grown in a greenhouse and photographs were taken 16 days after inoculation.

the $F$. graminearum spore suspension was sprayed onto wheat heads with the BN1 spore suspension, the disease severity of FHB was significantly reduced compared to that of FHB caused by only $F$. graminearum inoculation. However, when the wheat heads were inoculated by the pointinoculation method, BN1 did not have any efficacy against F. graminearum (Fig. 3 and Table 1).

\section{Discussion}

Epidemics of FHB have re-emerged worldwide (Xu and Nicholson, 2009), and frequent rainfall during the wheat flowering season in 2011 in Korea resulted in FHB epidemics and fatal yield losses in wheat production. Even after reducing the light wheat kernels that might be infected by $F$. graminearum, germination rate of wheat seeds was below $70 \%$, resulting in a shortage of seeds for planting that year. In spite of the drastic yield losses in wheat production, there are neither resistant cultivars against this disease nor registered fungicides for seed treatment and field application in Korea. These severe situations require the development of biological agents against FHB, and the present study provides one potential biological agent purified from a rice field.

Recently, the population structure of $F$. graminearum field strains in Korea have been extensively studied based on molecular markers and chemical analyses (Lee et al., 2009; 2010; 2012). The Fusarium species complex consists of at least 14 phylogenetic lineages based on the genealogical concordance (O'Donnell et al., 2000), and recently each lineage was classified as a new species (O'Donnell et al., 2004; 2008). The geographic location often influences the lineage distribution. For example, lineage 7 ( F. graminearum sensu stricto) is predominant in North America and lineage 6 ( $F$. asiaticum $)$ is predominant in Asian regions including Korea, China, and Japan (Gale et al., 2002; Lee et al., 2009; 2012; Suga et al., 2008). In Korea, the population structure is more complicated than that of other countries. In southern provinces of Korea, lineage 6 is predominant in rice and wheat. In the middle-eastern provinces, lineage 6 is predominant in rice fields, but lineage 7 is predominant in maize fields. Lineage 6 may be predominant in rice fields of Korea because it is better suited to a rice agro-ecosystem than the other lineages present in Korea (Lee et al., 2009). In addition, $F$. graminearum strains in Korean rice and maize fields have different genetic characteristics compared to those of other countries (Lee et al., 2009, 2012). The populations have lower genetic and genotypic diversity, suggesting that the populations have been maintained through asexual and homothallic events. These studies suggest the need to develop approaches to maintain the Korean $F$. graminearum populations.

Korea predominantly uses organic wheat production, and no chemical fungicides or biological agents have been registered for controlling wheat diseases or seed treatment. Therefore, controlling diseases in Korean wheat fields has focused on cultivation techniques, which have not been sufficient to control FHB epidemics. Frequent epidemics of FHB in Korea have led to the registration of fungicides such as prothioconazole, tebuconazole, and metconazole that have been widely used worldwide (Pirgozliev et al., 2003). However, frequent usage of the fungicides has tri- 
ggered the occurrence of $F$. graminearum strains resistant to the fungicides (Becher et al., 2010). The registration and application of fungicides in Korea could possibly control FHB in Korea for a few seasons, but fungicide-resistant strains will likely develop. Therefore, we need to develop alternative methods of control, including finding biological agents against $F$. graminearum.

Microorganisms have been used for development of biological agents against various plant pathogens, particularly the bacterial genus Streptomyces that has more than 600 species and produces various kinds of secondary metabolites with antagonistic effects to other microbes (http:// www.bacterio.cict.fr/s/streptomycesa.html). In this study, a new Streptomyces species was isolated from a Korean rice field and the strain showed a highly antagonistic effect against $F$. graminearum both in vitro and in vivo. The sequences of $16 \mathrm{~S}$ rRNA genes in the genus Streptomyces are highly conserved and are only useful for classification at the genus but not species level. Sequencing analysis showed that the 16S rRNA gene sequence of the BN1 strain matched morphological characteristics belonging to the genus Streptomyces. The ITS sequence of BN1 strain was different from those of known Streptomyces species. The phylogenetic tree showed that the ITS sequence of BN1 grouped with that of $S$. sampsonii with $77 \% \mathrm{BS}$, suggesting that BN1 does not belong to any known Streptomyces species. To classify the BN1 strain, further studies in morphological characteristics, phylogenetic analyses, and serological studies are needed.

The initial infection of $F$. graminearum occurs by ascospores discharged from perithecia during the short period (10-20 days) from anthesis through the soft dough stage of kernel development (Schroeder and Christensen, 1963). Infections are most serious when the anthers are exposed to $25-30^{\circ} \mathrm{C}$ with high humidity during flowering (Parry et al., 1995; Sutton, 1982). To verify the efficacy of the BN1 strain against $F$. graminearum, we directly inoculated wheat heads with spore suspension during mid-anthesis and maintained humidity for 2 days. Both spraying and point inoculation successfully caused FHB symptoms in wheat heads. However, the BN1 strain only efficiently antagonized $F$. graminearum with the spraying method, possibly because of either colonization of the BN1 strain within anthers or movement of the BN1 strain between anthers. Spores of F. graminearum germinate within anthers and move to another anther to cause blight symptoms throughout wheat heads. The BN1 strain did not trigger any disease symptom in wheat heads, suggesting that it is saprophytic on wheat plants, and the colonization of BN1 may be later than that of $F$. graminearum within a limited area such as wheat anthers. Also, the mycelial growth of $F$. graminearum is faster than that of $\mathrm{BN} 1$, and $F$. gramineruam efficiently overcomes the efficacy of BN1. Importantly, BN1 had an antagonistic effect using the spraying inoculation method, which is more natural than the point inoculation method for FHB development in wheat heads. This result suggests that the BN1 strain grew better on the wheat heads than in anthers, and that wheat heads can be successfully protected from $F$. graminearum infection by spraying BN1 spores during flowering.

In this study, a Streptomyces species strain BN1 was isolated from a rice field in Korea. The BN1 strain was identified as a new species based on the phylogenetic analysis of the ITS sequence. The efficacy of the BN1 strain against $F$. graminearum was characterized both in vitro and in vivo. BN1 efficiently inhibited the mycelial growth of $F$. gramienarum on media, and co-inoculation of $F$. graminearum and BN1 spore suspension into wheat heads reduced FHB disease severity. This study provides one of the best potential strategies for FHB control in Korea that could guarantee sustainable wheat production in Korea. Further studies are needed on the classification of BN1 at the species level and the mechanism of BN1 inhibition against $F$. graminearum.

\section{Acknowledgments}

This work was supported by the Dong-A University research fund.

\section{References}

Becher, R., Hettwer, U., Karlovsky, P., Deising, H. B. and Wirsel, G. R. 2010. Adaptation of Fusarium graminearum to tebuconazole yielded descendants diverging for levels of fitness, fungicide resistance, virulence, and mycotoxin production. Phytopathology 100:444-453.

Borneman, J. and Triplett, E. W. 1997. Molecular microbial diversity in soils from eastern Amazonia: evidence for unusual microorganisms and microbial population shifts associated with deforestation. Appl. Environ. Microbiol. 63:2647-2653.

Buchenauer, H. 1987. Mechanism of action of triazolyl fungicides and related compounds. In: Modern selective fungicides, ed. by H. Lyr, p. 205-232. Gustav-Fischer-Verlag, Jena, Germany.

Bujold, I., Paulitz, T. C. and Carisse, O. 2001. Effect of Microsphaeropsis sp. On the production of perithecia and ascospores of Gibberella zeae. Plant Dis. 85:977-984.

Capellini, R. A. and Peterson, J. L. 1965. Macroconidium formation in submerged cultures by a nonsporulating strain of Gibberella zeae. Mycologia 57:962-966.

Chan, Y. K., McCormick, W. A. and Seifert, K. A. 2003. Characterization of an antifungal soil bacterium and its antagonistic activities against Fusarium species. Can. J. Microbiol. 49: 253-262.

Dawson, W. A., Jestoi, M., Rizzo, A., Nicholson, P. and Bateman, G. L. 2004. Field evaluation of fungal competitors of Fusar- 
ium culmorum and Fusarium graminearum, causal agents of ear blight of winter wheat, for the control of mycotoxin production in grain. Biocontrol Sci. Technol. 14:783-799.

Desjardins, A. E. 2006. Fusarium mycotoxins: Chemistry, genetics and biology. APS Press, St. Paul, MN, USA.

Gale, L. R., Chen, L. F., Hernick, C. A. and Kistler, H. C. 2002. Population analysis of Fusarium graminearum from wheat fields in eastern China. Phytopathology 92:1315-1322.

Harris, S. D. 2005. Morphogenesis in germinating Fusarium graminearum macroconidia. Mycologia 97:880-887.

Lee, J., Chang, I., Kim, H., Yun, S.-H., Leslie, J. F. and Lee, Y.-W. 2009. Genetic diversity and fitness of Fusarium graminearum populations from rice in Korea. Appl. Environ. Microbiol. 75:3289-3295.

Lee, J., Kim, H., Jeon, J.-J., Kim, H-S., Zeller, K. A., Carter, L. L. A., Leslie, J. F. and Lee, Y.-W. 2012. Population structure and mycotoxin production of Fusarium graminearum from maize in Korea. Appl. Environ. Microbiol. 78:2161-2167.

Lee, S.-H., Lee, J., Nam, Y. J., Lee, S., Ryu, J.-G. and Lee, T. 2010. Population structure of Fusarium graminearum from maize and rice in 2009 in Korea. Plant Pathol. J. 26:321-327.

Leslie, J. F. and Summerell, B. A. 2006. The Fusarium laboratory manual. Blackwell Professional, Ames, IA, USA.

Luz, W. C. da, Stockwell, C. A. and Bergstrom, G. C. 2003. Biological control of Fusarium graminearum. In: Fusarium head blight of wheat and barley. eds. by K. J. Leonard and W. R. Bushnell. APS Press, St. Paul, MN, USA. pp. 381-394.

Normand, P., Ponsonnet, C., Nesme, X., Neyra, M. and Simonet, P. 1996. ITS analysis of prokaryotes. In: Molecular microbial ecology manual. eds. by D. L. Akkermans, J. D. Elsas, E. I. Bruijn. pp. 1-12. Kluwer Academic Publishers, Amsterdam, The Netherlands.

Nourozian, J., Etebarian, H. R. and Khodakaramian, G. 2006. Biological control of Fusarium graminearum on wheat by antagonistic bacteria. Songklanakarin J. Sci. Technol. 28:29-38.

O'Donnell, K., Kistler, H. C., Tacke, B. K. and Casper, H. H. 2000. Gene genealogies reveal global phylogeographic structure and reproductive isolation among lineages of Fusarium graminearum, the fungus causing wheat scab. Proc. Natl. Acad. Sci. USA 97:7905-7910.

O'Donnell, K., Ward, T. J., Aberra, D., Kistler, H. C., Aoki, T., Orwig, N., Kimura, M., Bjornstad, Å. and Klemsdal, S. S. 2008. Multilocus genotyping and molecular phylogenetics resolve a novel head blight pathogen within the Fusarium graminearum species complex from Ethiopia. Fungal Genet. Biol. 45:1514-1522.

O’Donnell, K., Ward, T. J., Geiser, D. M., Kistler, H. C. and Aoki, T. 2004. Genealogical concordance between the mating type locus and seven other nuclear genes supports formal recognition of nine phylogenetically distinct species within the Fusarium graminearum clade. Fungal Genet. Biol. 41:600-623.

Parry, D. W., Jenkinson, P. and McLeod, L. 1995. Fusarium ear blight (scab) in small grains. Plant Pathol. 44:207-238.

Pirgozliev, S. R., Edwards, S. G., Hare, M. C. and Jenkinson, P. 2003. Strategies for the control of Fusarium head blight in cereals. Eur. J. Plant Pathol. 109:731-742.
Pryor, S. W., Siebert, K., Gibson, D. M., Gossett, J. M. and Walker, L. P. 2007. Modeling production of antifungal compounds and their role in biocontrol inhibitory activity. J. Agric. Food Chem. 55:9530-9536.

Ryu, J.-G., Lee, S., Lee, S.-H., Son, S.-W., Nam, Y. J., Kim, M., Lee, T. and Yun, J.-C. 2011. Natural occurrence of Fusarium head blight and its mycotoxins in 2010-harvested barley and wheat grains in Korea. Res. Plant Dis. 17:272-279.

Saitou, N. and Nei, M. 1987. The neighbor-joining method: A new method for reconstructing phylogenetic trees. Mol. Biol. Evol. 4:406-425.

Sambrook, J. S. and Russell, D. 2001. Molecular cloning: A laboratory manual, Third ed. Cold Spring Harbor Laboratory Press, Cold Spring Harbor, NY, USA.

Schroeder, H. W. and Christensen, J. J. 1963. Factors affecting resistance of wheat scab caused by Gibberella zeae. Phytopathology 53:831-838.

Stockwell, C. A., Bergstrom, G. C. and Luz, W. C. da. 2001. Biological control of Fusarium head blight with Bacillus subtilis TrigoCor 1448. In: Proceedings of the 2001 National Fusarium Head Bight Forum, 8-10 Dec. 2001, Erlanger, Ky. Eds. by S. M. Canty, J. Lewis, L. Siler, and R. W. Ward. Michigan State University, East Lansing, MI, USA. pp. 91-95.

Suga, H., Karugia, G. W., Ward, T., Gale, L. R., Tomimura, K. Nakajima, T., Miyasaka, A. Koizumi, S., Kageyama, K. and Hyakumachi, M. 2008. Molecular characterization of the Fusarium graminearum species complex in Japan. Phytopathology 98:159-166.

Sutton, J. C. 1982. Epidemiology of wheat head blight and maize ear rot caused by Fusarium graminearum. Can. J. Plant Pathol. 4:195-209.

Tamura, K., Dudley, J., Nei, M. and Kumar, S. 2007. MEGA4: Molecular evolutionary genetics analysis (MEGA) software version 4.0. Mol. Biol. Evol. 24:1596-1599.

Ventura, M., Canchaya, C., Tauch, A., Chandra, G., Fitzgerald, G. F., Chater, K. F. and Sinderen, D. 2007. Genomics of Actinobacteria: tracing the evolutionary history of an ancient phylum. Microbiol. Mol. Biol. Rev. 71:495-548.

Watve, M. G., Tickoo, R., Jog, M. M. and Bhole, B. D. 2001. How many antibiotics are produced by the genus Streptomyces? Arch. Microbiol. 176:386-390.

Xu, X. and Nicholson, P. 2009. Community ecology of fungal pathogens causing wheat head blight. Annu. Rev. Phytopathol. 47:83-103.

Xue, A. G., Voldeng, H. D., Savard, M. E., Fedak, G., Tian, X. and Hsiang, T. 2009. Biological control of Fusarium head of wheat with Clonostachys rosea strain ACM941. Can. J. Plant Pathol. 31:169-179.

Yuen, G., Jochum, C. C., Osborne, L. E. and Jin, Y. 2003. Biocontrol of Fusarium head blight in wheat by Lysobacter enzymogenes strain C3. Phytopathology 93:S93.

Zhang, S., Schisler, D. A., Boehm, M. J. and Slininger, S. J. 2005. Carbon-to-nitrogen ratio and carbon loading of production media influence freeze-drying survival and biocontrol efficacy of Cryptococcus nodaensis $\mathrm{OH}$ 182.9. Phytopathology 95: 626-631. 